\title{
MRI Evaluation of Complete Response of Locally Advanced Rectal Cancer After Neoadjuvant Therapy: Current Status and Future Trends
}

\author{
Qiaoyu $X u^{\prime}$ \\ Yanyan $\mathrm{Xu}^{2}$ \\ Hongliang Sun $\mathbb{D D}^{2}$ \\ Tao Jiang' \\ Sheng Xie ${ }^{2}$ \\ Bee Yen Ooi ${ }^{3}$ \\ Yi Ding $\mathbb{D}^{\prime}$
}

'Department of Radiology, Beijing ChaoYang Hospital, Capital Medical University, Beijing, People's Republic of China; ${ }^{2}$ Department of Radiology, China-Japan Friendship Hospital, Beijing, People's Republic of China; ${ }^{3}$ Department of Radiology, Hospital Seberang Jaya, Penang, Malaysia

\begin{abstract}
Complete tumor response can be achieved in a certain proportion of patients with locally advanced rectal cancer, who achieve maximal response to neoadjuvant therapy (NAT). For these patients, a watch-and-wait (WW) or nonsurgical strategy has been proposed and is becoming widely practiced in order to avoid unnecessary surgical complications. Therefore, a non-invasive, reliable diagnostic tool for accurately evaluating complete tumor response is needed. Magnetic resonance imaging (MRI) plays a crucial role in both primary staging and restaging tumor response to NAT in rectal cancer without relying on resected specimen. In recent years, numerous efforts have been made to research the value of MRI in predicting and evaluating complete response in rectal cancer. Current MRI evaluation is mainly based on morphological and functional images. Morphologic MRI yields high soft tissue resolution, multiplanar images, and provides detailed depictions of rectal cancer and its surrounding structures. Functional MRI may help to distinguish residual tumor from fibrosis, therefore improving the diagnostic performance of morphologic MRI in identifying complete tumor response. Both morphologic and functional MRI have several promising parameters that may help accurately evaluate and/or predict complete response of rectal cancer. However, these parameters still have limitations and the results remain inconsistent. Recent development of new techniques, such as textural analysis, radiomics analysis and deep learning, demonstrate great potential based on MRI-derived parameters. This article aimed to review and help better understand the strengths, limitations, and future trends of these MRI-derived methods in evaluating complete response in rectal cancer.
\end{abstract}

Keywords: locally advanced rectal cancer, complete response, neoadjuvant therapy, magnetic resonance imaging

\section{Introduction}

Department of Radiology, China-Japan Friendship Hospital, No. 2 Yinghua East

Street, Chaoyang District, Beijing,

100029, People's Republic of China

Tel +86 10842055 I4

Fax +86 1064222963

Email stentorsun@gmail.com

Tao Jiang

Department of Radiology, Beijing ChaoYang Hospital, Capital Medical University,

No. 8 Gong-Ti South Road, Chaoyang

District, Beijing, 100020, People's

Republic of China

Tel +86 I37I8875778

Email jiangt8166@hotmail.com
Neoadjuvant therapy (NAT) has become a necessary part of the current standard of care for locally advanced rectal cancer (LARC) patients in downstaging the primary tumor and decreasing the risk of local tumor recurrence after surgical resection and improving prognosis. ${ }^{1,2}$ With the application of different NAT regimens, pathological complete response (pCR) may occur in approximately $10-30 \%$ of LARC patients. ${ }^{3-8}$ For these patients, surgical resection may not lead to an increase in overall and disease-free survival, but may result in extensive complications, such as sexual, urinary, and bowel dysfunction, and even increase the risk of anastomotic leak in patients with pCR. ${ }^{9}$

A watch-and-wait (WW) strategy has been proposed for patients who have a good response to NAT without residual tumor by clinical, endoscopic, and radiological 
surveillance, known as clinical complete response (cCR). WW strategy includes a series of follow-ups with surveillance examinations and salvage surgery for tumor recurrence. Because of its high rate of organ preservation and the equivalence of oncological outcomes in most of the patients, WW strategy has become widely practiced. ${ }^{3}$ The evaluation of $\mathrm{cCR}$ does not rely on a pathological examination of the resected specimen and is different from the evaluation of pCR. Thus, non-invasive tools which do not rely on the resected specimen to evaluate cCR accurately are needed.

Magnetic resonance imaging (MRI) plays a crucial role in both primary staging and restaging after NAT in rectal cancer. ${ }^{10,11}$ Because of its high soft tissue resolution and multiplanar imaging, MRI can provide a detailed depiction of rectal cancer and its surrounding structures. ${ }^{12}$ Moreover, the functional imaging and post-processing techniques of MRI allow quantitative analysis of residual tumors. ${ }^{13,14}$ The serial MRI monitoring during and after treatment can help evaluate LARC response to therapy, in order to schedule the NAT regimen in a timely manner. ${ }^{15}$ Thus, radiologists should recognize the importance of evaluating complete response of LARC and increase their diagnostic experience in this regard.

In recent years, numerous efforts have been made to research the value of MRI in predicting and evaluating complete response in LARC. This article aimed to review and help better understand the strengths, limitations, and future trends of these methods.

\section{Morphologic MRI}

\section{Volumetry}

Morphologic measurements of MRI are relatively easy and convenient in clinical practice. The most effective morphologic parameter is whole tumor volume (WTV), which is the sum of each cross-section area multiplied by section thickness based on the assessment of high resolution T2 weighted imaging (HR-T2WI). WTV analysis might improve the assessment of tumors by capturing inherent intratumoral heterogeneity, thus avoiding subjective judgment of different observers.

Several studies have investigated the value of WTV in assessing pCR of rectal cancer with relatively high accuracy, high specificity, and good reproducibility. ${ }^{5,6}$ The preNAT tumor volume does not seem to be effective in predicting $\mathrm{pCR}$ in most studies. The post-treatment and the percentage reduction of WTV based on MRI are good parameters for evaluating complete tumor responce. ${ }^{4-6,16-18}$

Furthermore, several studies found that tumor shrinkage mainly occurred shortly after the beginning of NAT, and the early tumor volume reduction rate after NAT may offer useful early information to predict the treatment outcomes in rectal cancer. ${ }^{6,19}$ Palmisano et al found that the combined assessment of $\operatorname{Vmid}\left(<4.8 \mathrm{~cm}^{3}\right)$ and $\Delta V$ mid appears effective in predicting pCR early with good accuracy $(81.5 \%)$, where Vmid and $\Delta$ Vmid were the cancer volume at mid-MRI and the percentage of volume reduction at mid-MRI. ${ }^{6}$

The efficacy of WTV in predicting complete tumor response may be due to better reproducibility by involving information of the whole tumor in comparison to 1-dimensional and 2-dimensional predictors. Volumetric analysis based on HR-T2WI can help predict complete tumor response with relatively high accuracy and specificity. However, WTV analysis is time-consuming, which limits its clinical practice. The development of automated

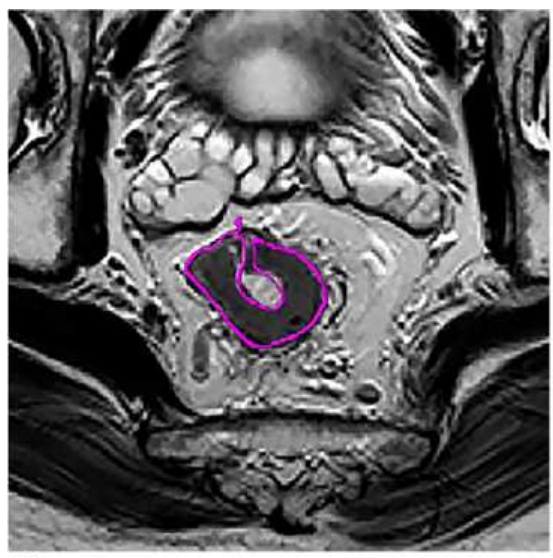

A

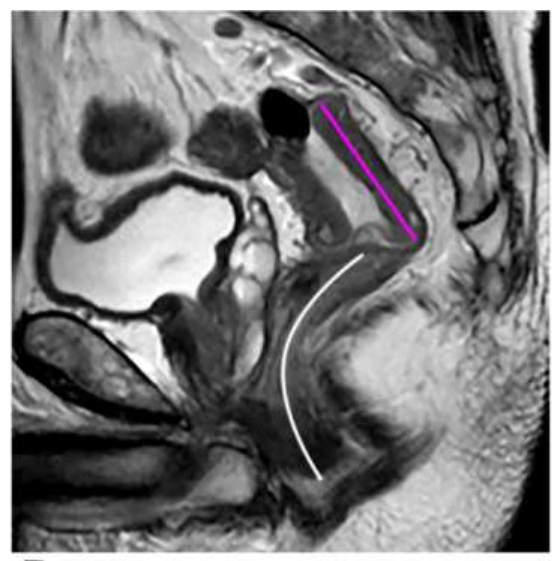

B

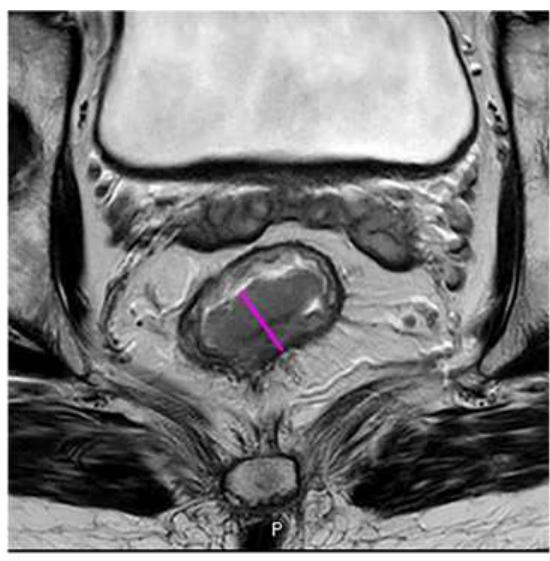

C

Figure I A 55-year-old man with LARC. (A) The maximum tumor area (MTA, pink curved line) on oblique axial HR-T2WI. (B) The maximum tumor length (MTL, pink straight line) and the distance from tumor to anal verge (DTA, white curved line) on sagittal T2WI. (C) The maximum tumor thickness (MTT, pink straight line) on oblique axial HR-T2WI. 
segmentation techniques may contribute to more efficient tumor analysis for better clinical application, but it still needs further research.

\section{Morphologic Predictors: I/2/}

\section{3-Dimensional Parameters}

Several convenient morphologic parameters have also been proven useful in assessing pCR, such as the lower maximum tumor area (MTA), lower maximum tumor length (MTL), cylindrical approximated tumor volume $($ CATV: MTA $\times$ MTL) and the higher reduction rates of
MTA, MTL, maximum tumor thickness (MTT), and CATV after NAT (Figure 1). Zhang et al found the reduction rates of MTA had the highest area under the curve (0.853) among these morphologic parameters in assessing the pCR response to NAT. ${ }^{4}$

The value of distance to anal verge (DTA) in pCR remained controversial. Zhang et al found the smaller DTA predicted a greater likelihood of pCR (Figure 1). ${ }^{4}$ Some studies also showed that a distance from anal verge smaller than 5 or $6 \mathrm{~cm}$ correlated with a favorable response. ${ }^{20-22}$ Low rectal cancer may gain more benefits from radiation therapy because of its lower mobility,

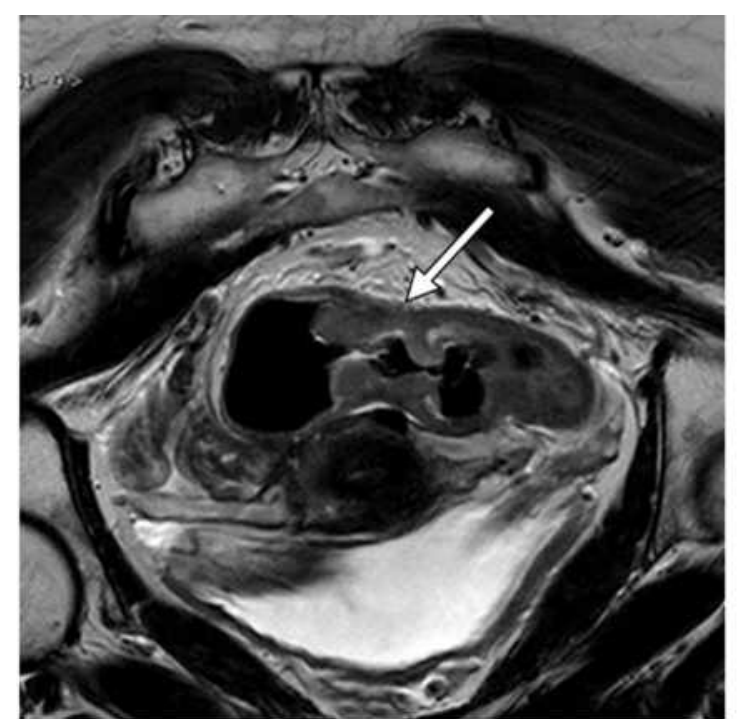

$\bar{A}$

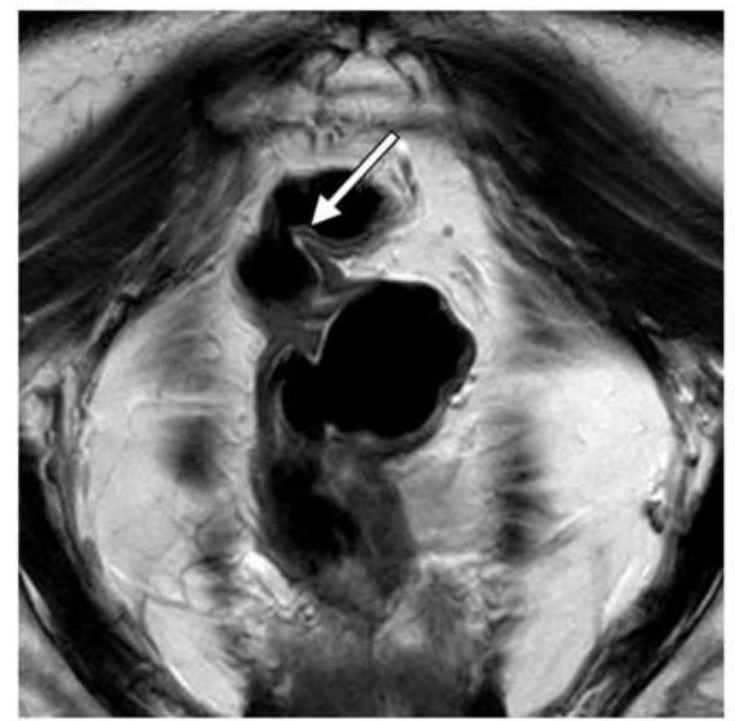

C

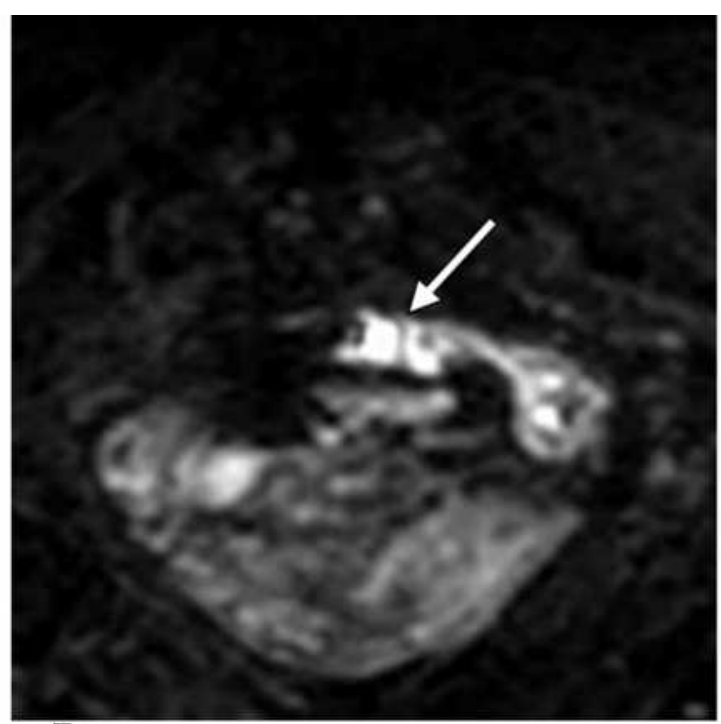

B

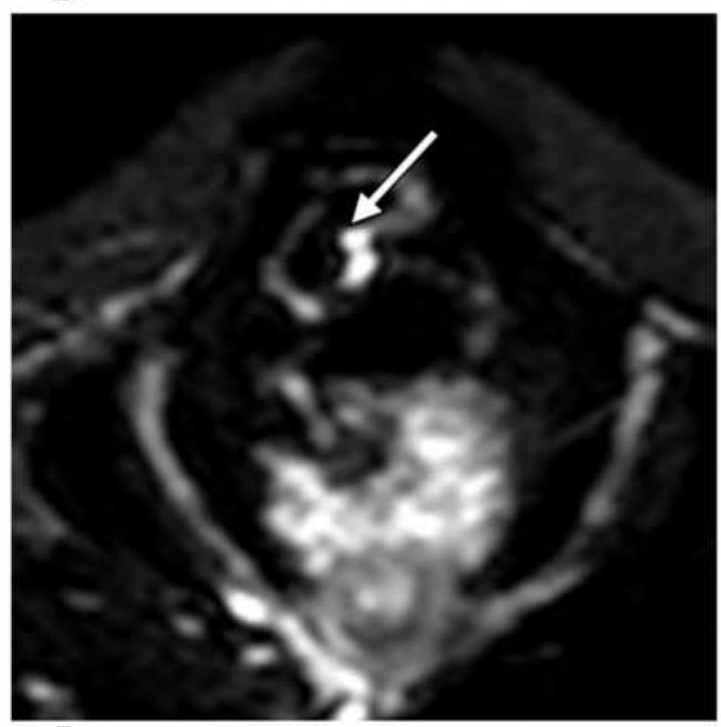

D

Figure 2 A 62-year-old woman with LARC. The oblique coronal T2WI (A) and DWI $\left(b=1000 \mathrm{~s} / \mathrm{mm}^{2}\right)$ (B) show primary rectal cancer before therapy (white arrow). Six weeks after the end of NAT, uneven thickening of the intestinal wall was still shown with intermediate signal intensity in oblique coronal T2WI (C) and hyperintensity signal intensity in DWI $\left(b=1000 \mathrm{~s} / \mathrm{mm}^{2}\right.$ ) (D), which mimic residual tumor (white arrow). Six days later, the patient underwent total mesorectal excision (TME), and the surgical resection specimen achieved $P C R$. 
compared with mid and high rectal tumors. Nevertheless, there have been several studies with contradictory findings, ${ }^{23,24}$ and even Patel et $\mathrm{al}^{24}$ reported that the patients with low tumors $(<4 \mathrm{~cm})$ and higher tumors $(>8 \mathrm{~cm})$ were less likely to have a pCR.

Limitation: the evaluation of complete tumor response by morphologic MRI is mainly based on HR-T2WI, which still has a relatively high false-negative rate. This limitation is mainly due to treatment-induced tumor fibrosis and edema (Figure 2). The treatment-induced fibrosis appears as the intermediate signal intensity on T2WI mimicking residual tumor. Moreover, the treatment-induced edema shows high signal on T2WI and lead to rectal wall swelling. It is difficult to assess residual tumors mixed with edema in the intestinal wall. Furthermore, the diagnostic accuracy of mostly morphologic predictors is suboptimal in predicting complete tumor response. The assessment outcomes are highly dependent on the subjective judgment of different observers.

\section{DWI and DWI-Derived Parameters \\ Diffusion-Weighted Imaging (DWI)}

DWI is performed routinely and assessed qualitatively for restaging to evaluate response to CRT, with the capability to characterize the tumor's cellular environment. The combination of DWI and T2 HR-MRI (high-resolution MRI) can help improve sensitivity and inter-observer agreement in assessment of complete response of LARC compared with T2 HR-MRI alone. ${ }^{7}$ The T2 HR-MRI could help locate the site of lesions of the rectal, and DWI could help differentiate residual tumor from fibrosis. Bates et al also found the higher $b$ value of DWI may increase the sensitivity of detection of residual tumor after NAT. ${ }^{25}$ Sathyakumar et $\mathrm{al}^{26}$ and Lambregts et $\mathrm{al}^{27}$ found that the change of tumor volume after therapy on DWI could help to identify complete tumor response in rectal cancer.

Limitation: however, DWI still had a low specificity in identifying complete tumor response, ${ }^{28,29}$ which might

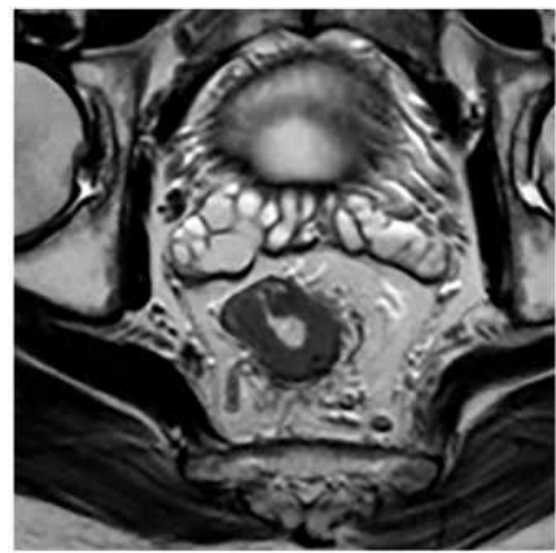

A

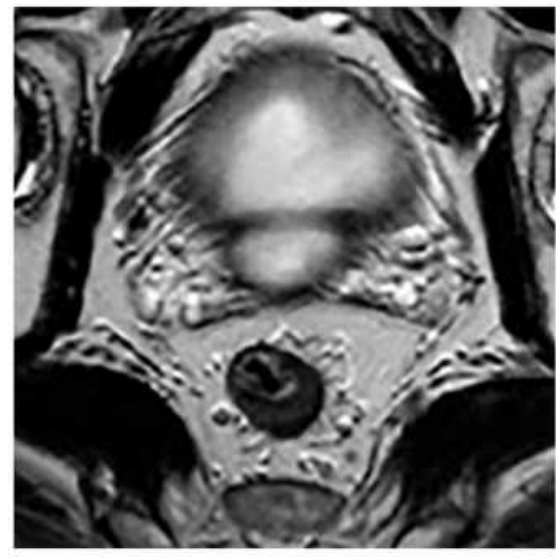

D

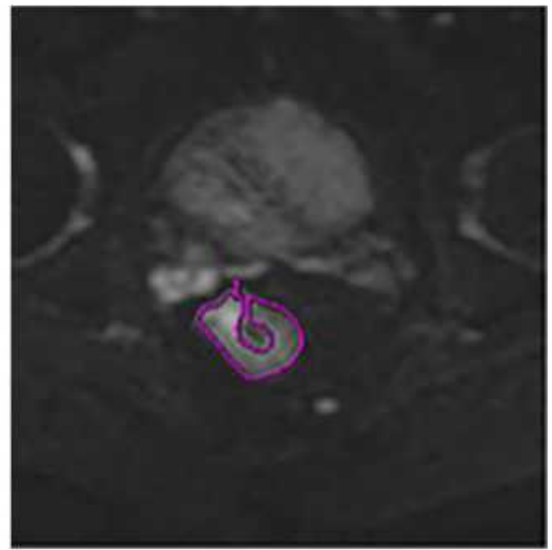

B

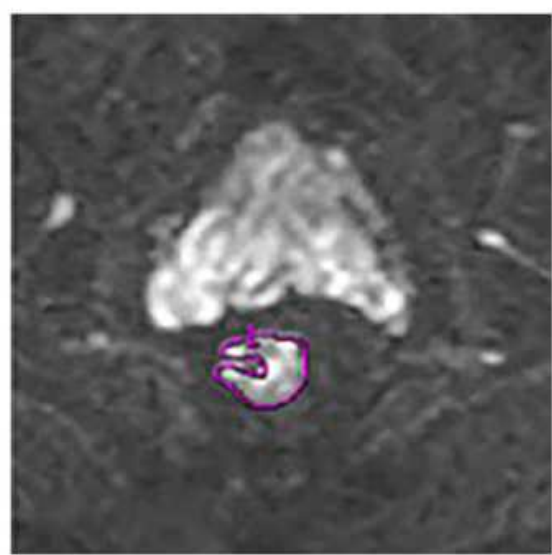

E

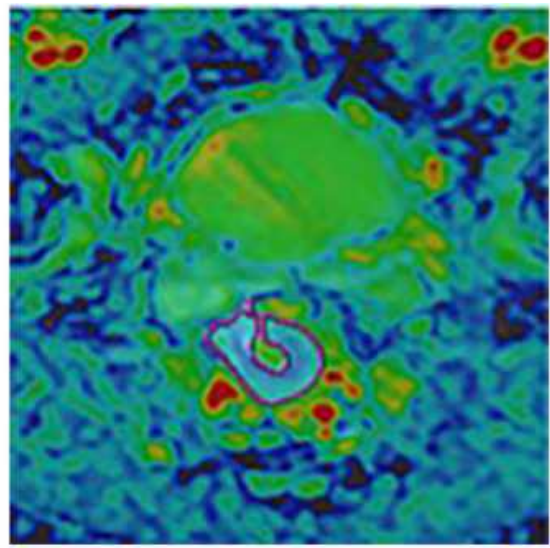

C

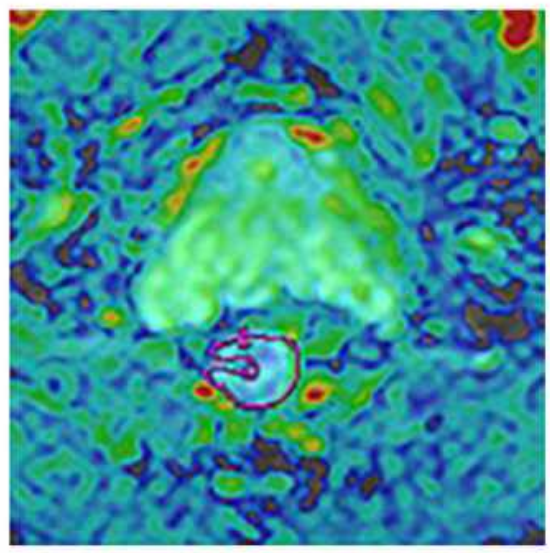

$\mathbf{F}$

Figure 3 A 55-year-old man with LARC who achieved pCR after therapy. The oblique axial HR-T2WI (A and D), oblique axial DWI (b = I000 s/mm $\left.{ }^{2}\right)(\mathbf{B}$ and $\mathbf{E})$, oblique axial ADC map $(\mathbf{C}$ and $\mathbf{F})$ show the images of LARC before $(\mathbf{A}-\mathbf{C})$ and after $(\mathbf{D}-\mathbf{F})$ NAT. The mean $A D C$ values were $0.87 \times 10^{-3} \mathrm{~mm}^{2} / \mathrm{s}(\mathbf{C}), 1.11 \times 10^{-3} \mathrm{~mm} / \mathrm{s}(\mathbf{F})$ respectively. 
lead to a relatively high level of false-negative rates (Figure 2). The image quality of DWI is vulnerable to geometric distortion and sensitivity to susceptibility artifacts. Several studies found that readout segmented echoplanar imaging (rs-EPI) may improve overall imaging quality and diagnostic performance compared to standard single-shot echo-planar imaging (ss-EPI) DWI in assessing complete response of $\mathrm{LARC},{ }^{28,30,31}$ but still needs to be proven in a large multicenter patient cohort. The preparation for the MRI scan is important for DWI, such as injecting spasmolytic medication to prevent intestinal peristalsis. Moreover, the optimal choice for DWI b values for assessment of complete tumor response has not been established, and further study is still needed.

\section{DWI-Derived ADC}

Apparent diffusion coefficient (ADC), the quantitative parameter of DWI, has been regarded as a reasonable imaging biomarker, which could quantitatively measure random diffusion of water molecules in biological tissues.
The decrease in tissue cellularity and the increase of extracellular space after therapy may increase ADC values (Figure 3).

Several studies have demonstrated the potential of $\mathrm{ADC}$ in distinguishing pCR from non-pCR. In most studies, the percentage changes of $\mathrm{ADC}$ values before and after NAT can help identify pCR of LARC, with better diagnostic performance than pre- and post-ADC values. . $28,32-35$ However, the results of pre-NAT and post-NAT ADC values remain very inconsistent. Several studies found lower pre-ADC values ${ }^{8,33,36,37}$ and higher post-ADC values ${ }^{28,32-34,36,38,39}$ in pCR group than nonpCR group, but several studies found no significant difference in pre-ADC values ${ }^{28,32,34,38-40}$ and post-ADC values $^{8,37,40,41}$ between these two groups. This discrepancy suggested that evaluation of treatment response may be limited when simply using pre- and post-ADC values.

Histogram parameters may help improve this issue and provide more reliable information on tumors. In histogram

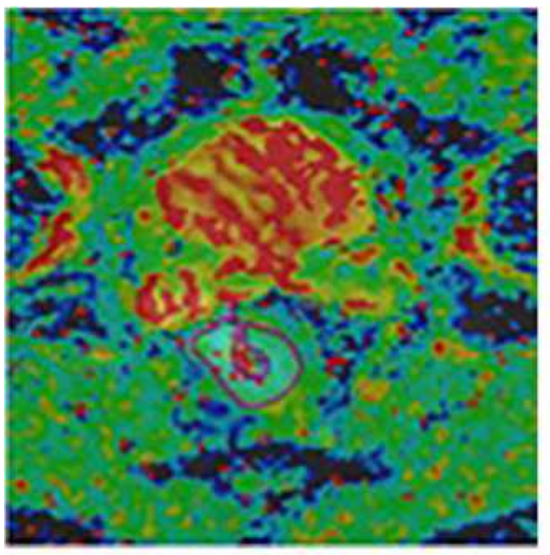

A

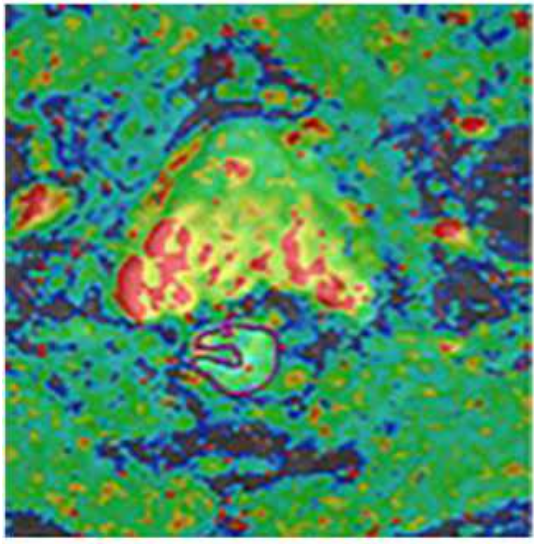

D

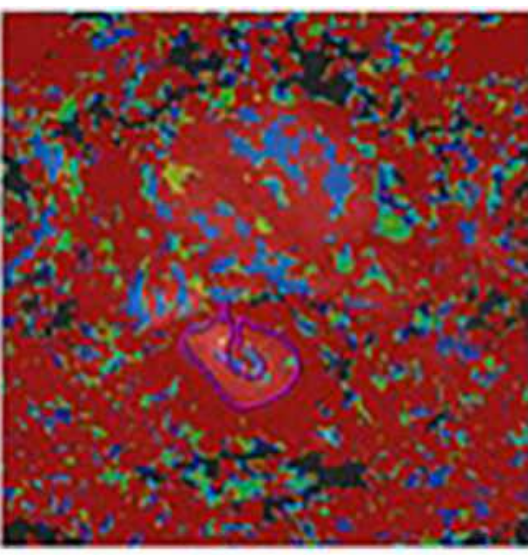

B

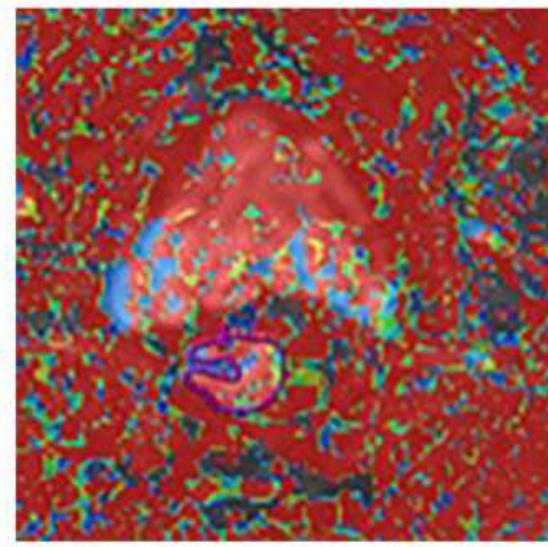

E

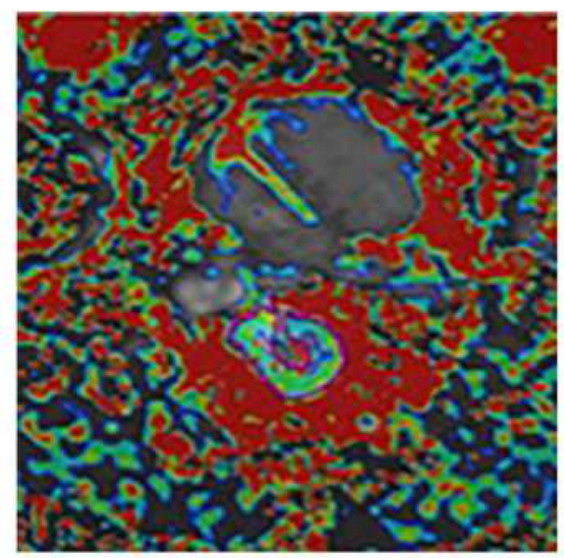

C

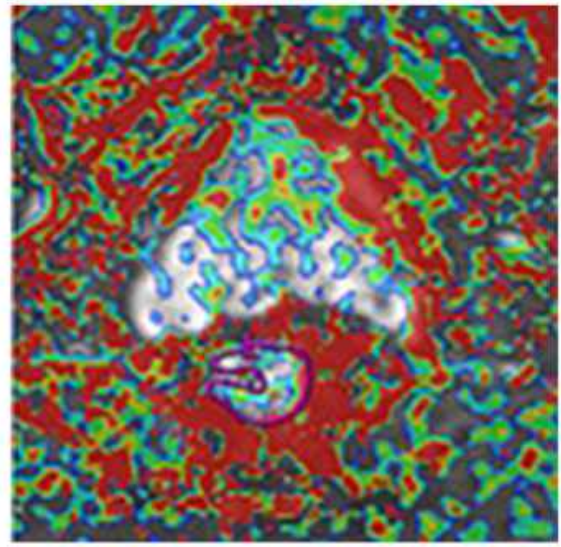

$\mathbf{F}$

Figure 4 A 55-year-old man with LARC who achieved pCR after therapy. IVIM parameters derived from DWI before (A-C) and after (D-F) NAT. (A and D) Oblique axial $D$ map; (B and $\mathbf{E})$ oblique axial $D *$ map; (C and $\mathbf{F})$ oblique axial $f$ map. 
analysis of ADC, Yang et $\mathrm{al}^{28}$ found the 95 th percentiles of post-NAT ADC value, and its percentage change showed a comparative good diagnostic performance and a higher specificity in assessing pCR. Choi et $\mathrm{al}^{30}$ showed that postNAT ADC histogram analysis with 25th percentiles had the best diagnostic performance for detecting $\mathrm{pCR}$. Enkhbaatar et $\mathrm{al}^{35}$ demonstrated that post-NAT skewness derived from the ADC histogram was a useful tool for predicting a favorable response to NAT.

Limitation: the discordant results may be because the ADC value could reflect the rate of diffusion in cellular tissues but failed to distinguish the perfusion effects from true tissue diffusion, thereby introducing the signal attenuation of imaging.

\section{DWI-Derived IVIM}

Intravoxel incoherent motion (IVIM) model, could distinguish perfusion information from diffusion using a biexponential model and multi-b-value sampling. Hence, the true molecular diffusion and microcirculation perfusion could be obtained simultaneously. ${ }^{42}$ The parameters of the IVIM model mainly include $\mathrm{D}, \mathrm{D}^{*}, f$ values. The $\mathrm{D}$ value is the diffusion coefficient of slow or nonperfusion-related diffusion, which represents true molecular diffusion, while $\mathrm{D}^{*}$ value is the diffusion coefficient of fast or perfusion-related diffusion, and $f$ (given as a percentage) is the perfusionrelated diffusion fraction, which represents fractional volume occupied in the voxel by flowing spins (Figure 4).

The most promising IVIM-derived parameter in predicting complete response of rectal cancer might be $\mathrm{D}$ values. The lower pre-D values, ${ }^{8,37}$ higher percentage changes of D values, ${ }^{8}$ and the higher post- $\mathrm{D}^{43}$ values have been found in pCR group compared with the non-pCR group. Conversely, several studies reported that pre-D values ${ }^{43,44}$ and post- $\mathrm{D}^{8,44}$ values were not associated with the pCR status.
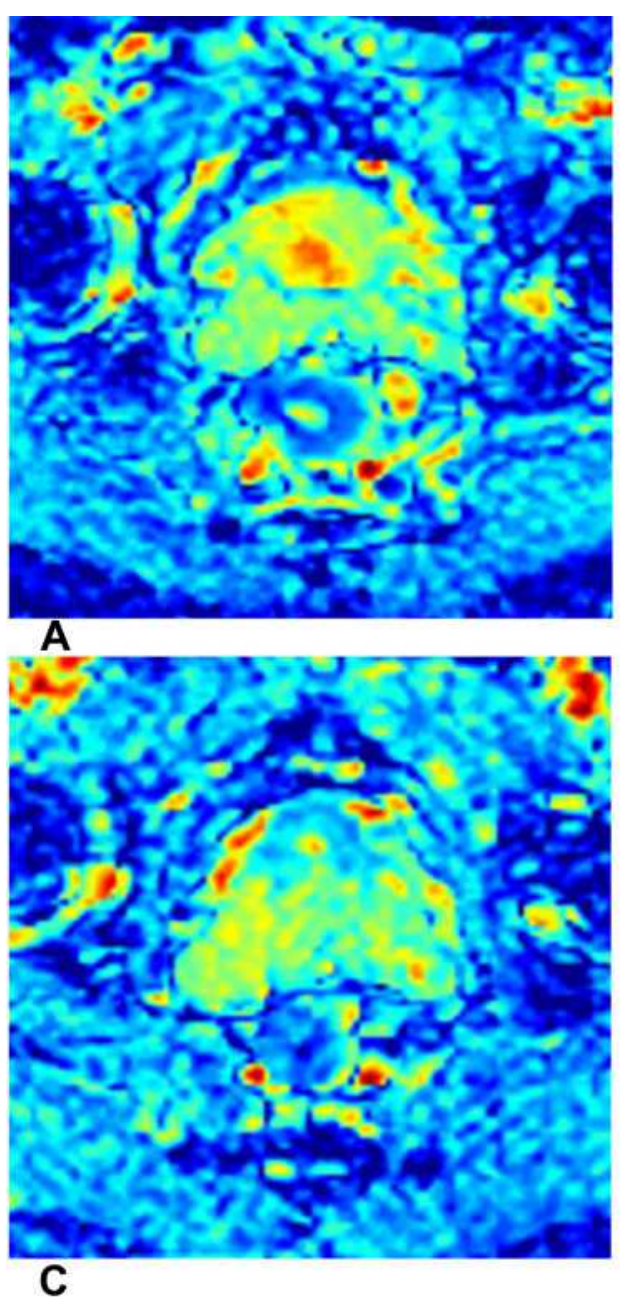

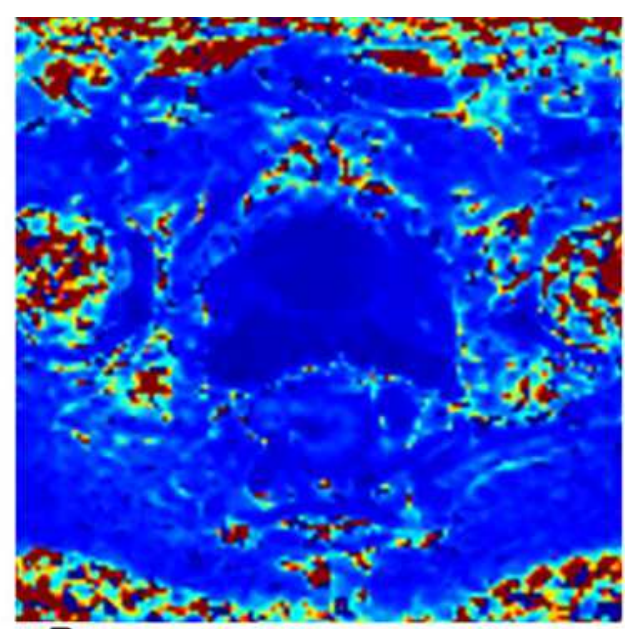

B

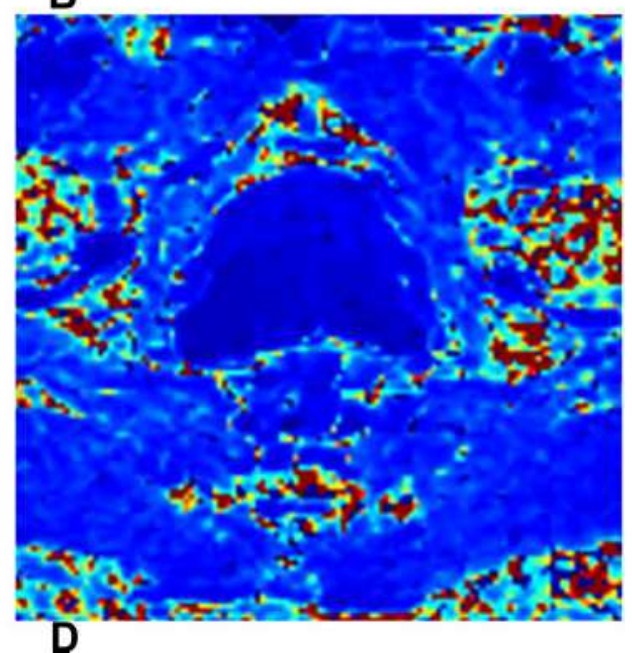

Figure 5 A 55-year-old man with LARC who achieved PCR after therapy. DKI parameters derived from DWI before (A and B) and after (C and D) NAT. (A and C) Oblique axial D map; (B and D) oblique axial $\mathrm{K}$ map. 
The results of $f$ values in assessing treatment response have been variable across different studies. The higher pre$f^{43}$ and higher post $-f^{8}$ were found in the pCR group of rectal cancer, whereas other studies found that $f$ value was not useful in assessing the treatment response in rectal tumor. ${ }^{44}$ These conflicting results might be because $f$ value represented the signal intensity ratio of blood capillaries and tumor tissues, which were both affected by the $\mathrm{T} 2$ contribution. After treatment, the T2 values of the tumor tended to increase, leading to an underestimation of the increase in $f$ value. Thus, the utility of $f$ is of limited significance in clinical practice.

The results of $\mathrm{D}^{*}$ values have also remained highly controversial, the higher pre- $\mathrm{D}^{*}$ values had been found in pCR group of rectal cancer in several studies, ${ }^{43,45}$ but other studies found $\mathrm{D}^{*}$ values were useless in predicting or identifying pCR response. ${ }^{8,44,46}$ These conflicting outcomes might be the result of the relatively high sensitivity of $\mathrm{D}^{*}$ values to noise leading to relatively poor reproducibility.
Limitations: the post-processing techniques of IVIM are relatively complex and time-consuming. IVIM parameters exhibit poor repeatability and reproducibility, especially for $\mathrm{D}^{*}$ and $f$ value. Several studies proved that whole-tumor volume measurement, ${ }^{8}$ histogram analysis ${ }^{37}$ may improve this problem and help obtain reliable results. But histogram analysis of IVIM still found the mean values were better than or equal to the histogram metrics. ${ }^{37,46}$ IVIM model required sufficient $b$ values, however, too many $b$ values would increase the scan time. Therefore, the proper number and interval of $b$ values need to be explored.

\section{DWI-Derived DKI}

Diffusion kurtosis imaging (DKI) is an advanced nonGaussian DWI model, which includes both diffusivity and kurtosis parameters. Diffusivity is the diffusion coefficient (D) with the correlation of non-Gaussian bias, whereas kurtosis coefficient $(\mathrm{K})$ measures the deviation of tissue diffusion from a Gaussian model. ${ }^{47}$ (Figure 5)

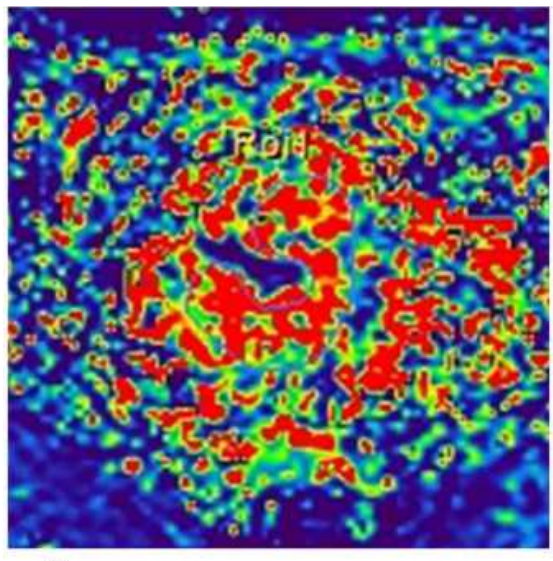

A

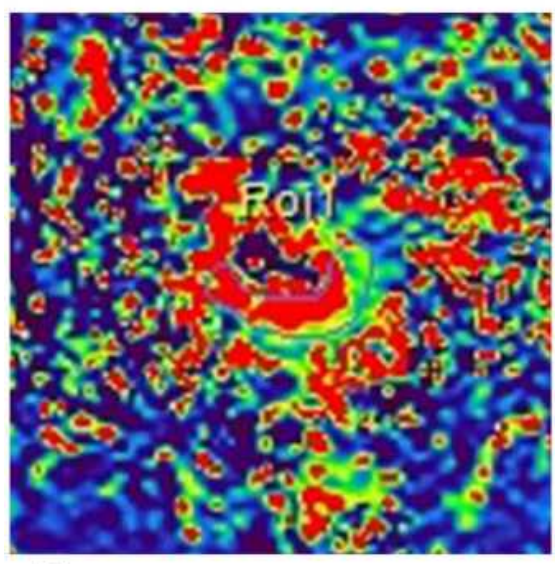

D

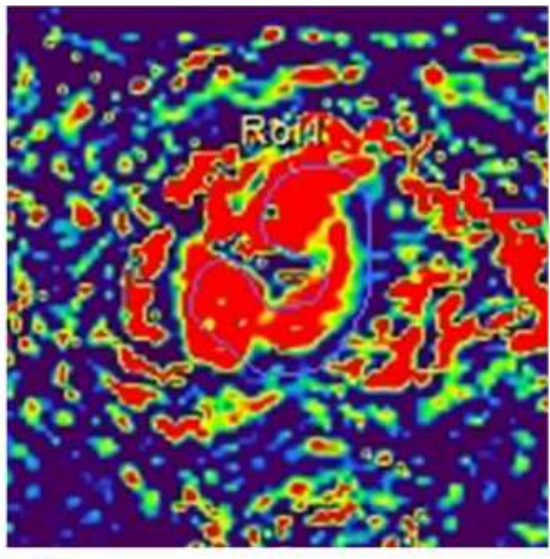

B

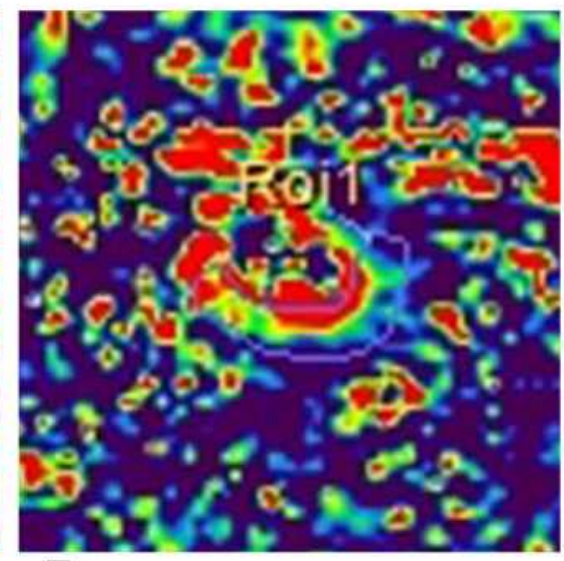

E

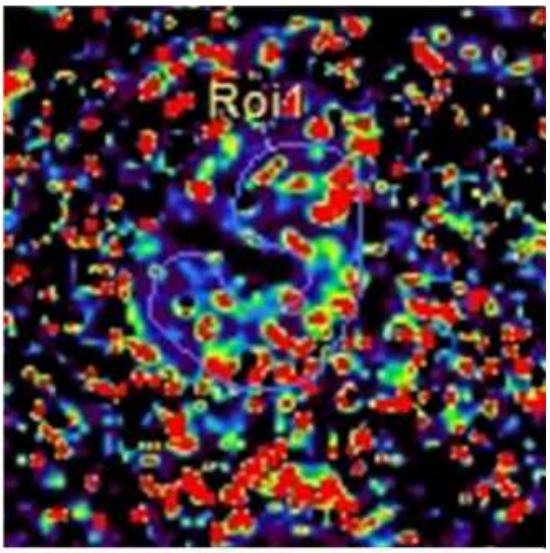

C

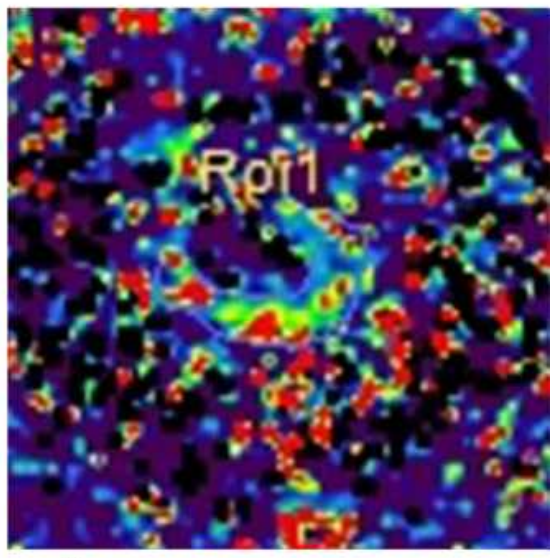

$\mathbf{F}$

Figure 6 A 6I-year-old man with LARC who achieved PCR after therapy. Dynamic Contrast-Enhanced MRI before (A-C) and after (D-F) neoadjuvant chemoradiation therapy for locally advanced rectal cancer which achieved pathological complete response. (A and $\mathbf{D})$ Oblique axial $\mathrm{K}^{\text {trans }}$ map; (B and $\left.\mathbf{E}\right)$ oblique axial $\mathrm{K}_{\mathrm{ep}}$ map; (C and $\mathbf{F}$ ) oblique axial $\mathrm{V}_{\mathrm{e}}$ map. 
$\mathrm{Hu}$ et $\mathrm{al}^{39}$ found lower pre-K, lower post-K, and higher post-D values in $\mathrm{pCR}$ group compared with non-pCR group, and the post-K showed higher specificity and specificity than conventional DWI for assessing pCR and nonpCR in patients with LARC. Furthermore, Yu et $\mathrm{al}^{48}$ found 10 th percentiles of pre-D values were significantly lower in the good responder group.

Limitations: only a few studies focused on the value of DKI in identifying complete tumor response, and the study scale was relatively small. The application of DKI technology needs higher $b$ values and more $b$ values, and the different combinations of $b$ values may result in different results. Thus, the value of DKI in identifying complete tumor response still needs further research.

\section{Dynamic Contrast-Enhanced-MRI}

Dynamic contrast-enhanced (DCE)-MRI can assess tissue perfusion and oxygenation at a macroscopic level. The results of DCE-derived parameters remain controversial among different studies. Several studies found the parameters of DCE, including $\mathrm{K}^{\text {trans }}$ (plasma-to-extravascular volume transfer), $\mathrm{K}_{\mathrm{ep}}$ (extravascular-to-plasma volume transfer constant), and $\mathrm{V}_{\mathrm{e}}$ (extravascular extracellular volume fraction per unit of tissue volume), $\mathrm{V}_{\mathrm{p}}$ (plasma volume), can help evaluate pCR for NAT in LARC.

The higher pre- $\mathrm{K},{ }^{\text {trans } 49}$ pre- $\mathrm{K}_{\mathrm{ep}},{ }^{50}$ pre- $\mathrm{V}_{\mathrm{e}}{ }^{50}$ values, lower post- $\mathrm{K}^{\text {trans51 }}$ values, and the higher changes of
$\mathrm{K}^{\text {trans }}$ values after $\mathrm{NAT}^{52}$ were found in the pCR group than non-pCR group of rectal cancer. However, Gollub et $\mathrm{al}^{53}$ still found DCE-derived parameters could not distinguish between pCR group and non-pCR group. Although DCE-derived parameters may have the potential to evaluate complete tumor response in LARC, the accuracy needs to be verified (Figure 6).

Thus, the quantitative parameters, such as ADC-, IVIM-, DKI- and DCE-derived parameters, might improve the diagnostic performance of conventional MRI and become promising predictors of complete response in rectal cancer, but the results have remained inconsistent and the post-processing techniques are relatively complex, time-consuming, and have poor repeatability.

\section{Challenges and Future Trends}

MRI evaluation of complete response in rectal cancer remains challenging. First, different MR scanning protocols, software, and equipment may induce controversial results, poor repeatability, and a variety in cut-off values. Secondly, the different study time points also influence the results in predicting and evaluating complete tumor response, such as the interval between each assessment time by MRI during NAT, the completion of NAT and restaging MRI, restaging MRI and surgery. Thirdly, the influence of neoadjuvant treatment protocol, inclusion criteria (such as mucinous adenocarcinoma, signet-ring cell

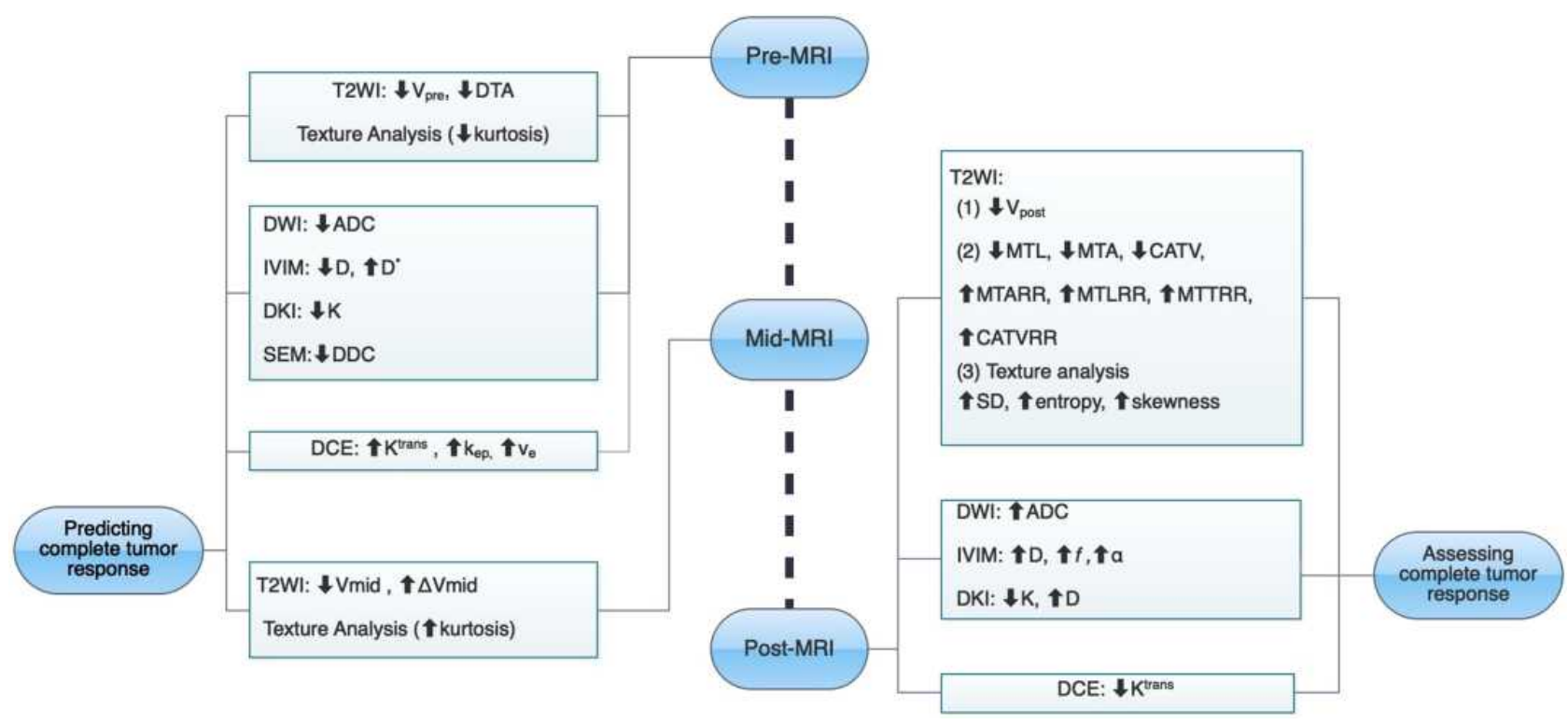

Figure 7 The summary chart of multiple MRI methods for predicting and assessing complete tumor response after neoadjuvant therapy in locally advanced rectal cancer. Abbreviations: CATV, cylindrical approximated tumor volume; CATVRR, cylindrical approximated tumor volume reduction rate; DTA, distance from tumor to anal verge; MTA, maximum tumor area; MTARR, maximum tumor area reduction rate; MTL, maximum tumor length; MTLRR, maximum tumor length reduction rate; MTT, maximum tumor thickness; MTTRR, maximum tumor thickness reduction rate; PCR, pathological complete response; V, volume. 
carcinoma) also need to be noted in MRI evaluation. Lastly, most studies were performed in a single institution on a small scale, so multi-centered research in an independent and large multicenter patient cohort is needed in further studies.

Recent advances in several new techniques have shown great potential for complete tumor response prediction and evaluation, such as MRI-derived textural analysis, radiomics analysis, and deep learning. Texture analysis (TA), an emerging area of radiomics, has been applied in rectal cancer recently. TA can objectively assess the heterogeneousness of the tumor. Several studies found TA parameters derived from T2WI, ${ }^{54-56} \mathrm{DWI},{ }^{56-58}$ and $\mathrm{DCE}^{52}$ can predict complete response in rectal cancer. Some studies indicated the radiomics analysis extracted high-dimensional imaging features of rectal cancer, rather than the relatively low dimensional

Table I Summary of Statistically Significant MR Parameters in Predicting and Evaluating Complete Tumor Response of Rectal Cancer After NAT

\begin{tabular}{|c|c|c|}
\hline First Author (Year) & Method & Statistically Significant MR Parameters \\
\hline Zhang $^{4}$ (2017) & $\mathrm{T} 2 \mathrm{Wl}$ & $\begin{array}{l}\downarrow M T A_{\text {post }}, \downarrow M T L_{\text {post }}, \downarrow D T A_{\text {pre }}, \downarrow C A T V_{\text {post }}, \uparrow M T A R R, \uparrow M T L R R, \uparrow M T T R R, \\
\uparrow C A T V R R\end{array}$ \\
\hline Palmisano ${ }^{6}(2018)$ & $\mathrm{T} 2 \mathrm{Wl}$ & $\downarrow \mathrm{V}_{\text {post }}, \downarrow \mathrm{V}_{\text {mid }} \uparrow \Delta \mathrm{V}_{\text {mid }}, \uparrow \Delta \mathrm{V}$ \\
\hline Sathyakumar ${ }^{26}(2016)$ & T2WI, DWI & $\downarrow \mathrm{V}_{\mathrm{pre}}, \uparrow \Delta \mathrm{V}$ \\
\hline $\begin{array}{l}\text { Lambregts }^{27}(2015), \text { Curvo-Semedo } \\
(2011)\end{array}$ & T2WI, DWI & $\downarrow \mathrm{V}_{\text {post }}, \uparrow \Delta \mathrm{V}$ \\
\hline De Cecco ${ }^{54}(2015)$ & T2WI, TA & $\downarrow$ kurtosis $_{\text {pre }}, \uparrow$ kurtosis $_{\text {mid }}$ \\
\hline Aker ${ }^{55}$ & T2WI, TA & $\uparrow \mathrm{SD}_{\text {post }}, \uparrow$ entropy $\mathrm{post}, \uparrow$ skewness $_{\text {post }}$ \\
\hline Yang $^{56}$ & T2WI, DWI, TA & $\uparrow A D C_{\text {post }}, \downarrow$ uniformity \\
\hline De Felice ${ }^{40}(2017)$ & DWI & $\uparrow A D C_{\text {mid }}$ \\
\hline $\begin{array}{l}\mathrm{Kim}^{32}(20 \mathrm{II}), \text { Blazic }^{34}(20 \mathrm{I} 6), \text { Yang }^{28} \\
(2020)\end{array}$ & DWI & $\uparrow A D C_{\text {post }}, \uparrow \triangle A D C$ \\
\hline Intven ${ }^{33}(2013)$ & DWI & $\downarrow A D C_{\text {pre }}, \uparrow A D C_{\text {post }}, \uparrow \triangle A D C$ \\
\hline Lambrecht $^{36}(2012)$ & DWI & $\downarrow \mathrm{ADC}_{\text {pre }}, \uparrow A D C_{\text {post }}, \uparrow \Delta \mathrm{ADC}, \uparrow \Delta \mathrm{V}$ \\
\hline $\mathrm{Xu}^{8}(2018)$ & DWI (IVIM) & $\downarrow \mathrm{ADC}_{\text {pre }}, \downarrow \mathrm{D}(\mathrm{IVIM})_{\text {pre }}, \uparrow f_{\text {post }}, \uparrow \Delta \mathrm{ADC}, \uparrow \Delta \mathrm{D}$ (IVIM), $\uparrow \Delta f$ \\
\hline $\mathrm{Lu}^{43}(2017)$ & DWI (IVIM) & $\uparrow D_{\text {pre }}^{*} \uparrow f_{\text {pre }}, \uparrow \mathrm{D}(\mathrm{IVIM})_{\text {post }}, \uparrow \Delta \mathrm{ADC}, \uparrow \Delta \mathrm{D}$ (IVIM) \\
\hline $\mathrm{Zhu}^{44}(2017)$ & $\begin{array}{l}\text { DWI (IVIM, } \\
\text { SEM) }\end{array}$ & $\uparrow A D C_{\text {post }}, \uparrow \alpha p o s t, \uparrow \Delta A D C, \uparrow \Delta \alpha$ \\
\hline Liang $^{37}$ (2019) & $\begin{array}{l}\text { DWI (IVIM, } \\
\text { SEM) }\end{array}$ & $\downarrow A D C_{\text {pre }}, \downarrow D D C_{\text {pre }}, \downarrow D(I V I M)$ pre \\
\hline $\mathrm{Hu}^{39}(2017)$ & DWI (DKI) & $\uparrow A D C_{\text {post }}, \uparrow \Delta A D C, \downarrow K_{\text {pre }}, \downarrow K_{\text {post }}, \uparrow \Delta D(D K I), \uparrow D(D K I)_{\text {post }}$, \\
\hline Ciolina $^{49}(2019)$ & DCE & $\uparrow K_{\text {pre }}^{\text {trans }} \uparrow V_{\text {e post }}$ \\
\hline Tong $^{50}(2015)$ & DCE & $\uparrow K_{\text {pre }}^{\text {trans }}, \uparrow K_{\text {ep pre }}, \uparrow V_{\text {e pre }}$ \\
\hline Zou $^{52}(2019)$ & DCE, TA & $\downarrow \mathrm{K}_{\text {post }}^{\text {trans }} \uparrow \Delta$ variance, $\uparrow \Delta$ entropy, $\uparrow \Delta$ correlation \\
\hline
\end{tabular}

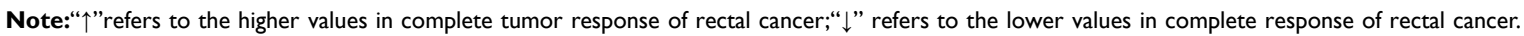

Abbreviations: V, volume; pre, before neoadjuvant therapy; mid, the middle of neoadjuvant therapy; post, after neoadjuvant therapy; $\Delta$ the percentage reduction; CATV, cylindrical approximated tumor volume; CATVRR, cylindrical approximated tumor volume reduction rate; DTA, distance from tumor to anal verge; MTA, maximum tumor area; MTARR, maximum tumor area reduction rate; MTL, maximum tumor length; MTLRR, maximum tumor length reduction rate; MTT, maximum tumor thickness; MTTRR, maximum tumor thickness reduction rate; ADC, apparent diffusion coefficient; IVIM, intravoxel incoherent motion; SEM, stretched exponential model; DDC, distributed diffusion coefficient; $\mathrm{D}(\mathrm{IVIM})$, slow diffusion coefficient; $\mathrm{D}^{*}$, fast diffusion coefficient; $f$, perfusion-related diffusion fraction; $\mathrm{D}$ (DKI), diffusion coefficient; $\mathrm{K}$, kurtosis coefficient; $\mathrm{DCE}$, dynamic contrast-enhanced; $\mathrm{K}^{\text {trans }}$, plasma-to-extravascular volume transfer; $\mathrm{K}_{\mathrm{ep}}$, extravascular-to-plasma volume transfer constant; $\mathrm{V}_{\mathrm{e}}$, extravascular extracellular volume fraction per unit of tissue volume; $V_{p}$, plasma volume; $T A$, texture analysis. 
features derived from texture analysis. Furthermore, several recent studies found the combination of the pre-treatment radiomics nomogram extracted from anatomical, diffusion, and perfusion images based on MRI can predict complete tumor response in patients with greater predictive efficacy than the utility of them alone in rectal cancer. ${ }^{59-61}$ The combination of the clinical features, MRI-derived methods, and other multiple imaging tools, which incorporate ${ }^{18}$ F-FDG PET/CT imaging also may achieve a greater diagnostic efficacy. ${ }^{62}$ Radiomics could predict response to therapy with diagnostic performance comparable to highly experienced radiologists, ${ }^{63}$ which indicates that radiomics has strong potential to be a decision-making tool in the clinical setting. Moreover, with the application of artificial intelligence, some recent studies found that the utility of deep learning models may enhance diagnostic performance for predicting complete response in rectal cancer. ${ }^{64,65}$ Nevertheless, the value of these methods has only been proven in a few studies, further strict and large multicenter research is still needed.

\section{Conclusion}

The evaluation of complete response is important in rectal cancer, and several MRI-derived methods are promising in predicting and identifying complete tumor response (Figure 7, Table 1). When considering patients with complete response in rectal cancer, strict criteria should be applied, especially the specificity of parameters. The misdiagnosis of complete tumor response may lead to tumor recurrence and poor prognosis. Although MRI is already the most used tool for evaluating rectal cancer, current MRI evaluation methods still have limitations. And the accurate evaluation of complete tumor response cannot be completely dependent on MRI alone currently. Researchers have found some promising MR parameters in assessing complete response of rectal cancer. And the development of new techniques, such as MRI-derived textural analysis, radiomics analysis, and deep learning, may enhance diagnostic performance, which incorporate the clinical features which may help MRI become a noninvasive and reliable decision-making tool in evaluating complete response of rectal cancer, but further exploration with larger sample sizes is still needed.

\section{Author Contributions}

All authors made substantial contributions to conception and design, acquisition of data, or analysis and interpretation of data; took part in drafting the article or revising it critically for important intellectual content; agreed to submit to the current journal; gave final approval of the version to be published; and agree to be accountable for all aspects of the work.

\section{Disclosure}

The authors report no conflicts of interest in this work.

\section{References}

1. Petrelli F, Trevisan F, Cabiddu M, et al. Total neoadjuvant therapy in rectal cancer: a systematic review and meta-analysis of treatment outcomes. Ann Surg. 2020;271(3):440-448. doi:10.1097/ SLA.0000000000003471

2. De Felice F, Benevento I, Magnante AL, et al. Clinical benefit of adding oxaliplatin to standard neoadjuvant chemoradiotherapy in locally advanced rectal cancer: a meta-analysis: oxaliplatin in neoadjuvant treatment for rectal cancer. BMC Cancer. 2017;17(1):325. doi:10.1186/s12885-017-3323-4

3. Smith JJ, Strombom P, Chow OS, et al. Assessment of a watch-andwait strategy for rectal cancer in patients with a complete response after neoadjuvant therapy. JAMA Oncol. 2019;5(4):e185896. doi:10.1001/jamaoncol.2018.5896

4. Zhang C, Ye F, Liu Y, Ouyang H, Zhao X, Zhang H. Morphologic predictors of pathological complete response to neoadjuvant chemoradiotherapy in locally advanced rectal cancer. Oncotarget. 2018;9 (4):4862-4874. doi:10.18632/oncotarget.23419

5. Martens MH, van Heeswijk MM, van den Broek JJ, et al. Prospective, multicenter validation study of magnetic resonance volumetry for response assessment after preoperative chemoradiation in rectal cancer: can the results in the literature be reproduced? Int J Radiat Oncol Biol Phys. 2015;93(5):1005-1014. doi:10.1016/j. ijrobp.2015.09.008

6. Palmisano A, Esposito A, Di Chiara A, et al. Could early tumour volume changes assessed on morphological MRI predict the response to chemoradiation therapy in locally-advanced rectal cancer? Clin Radiol. 2018;73(6):555-563. doi:10.1016/j.crad.2018.01.007

7. Chandramohan A, Siddiqi UM, Mittal R, et al. Diffusion weighted imaging improves diagnostic ability of MRI for determining complete response to neoadjuvant therapy in locally advanced rectal cancer. Eur $J$ Radiol Open. 2020;7:100223. doi:10.1016/j. ejro.2020.100223

8. Xu Q, Xu Y, Sun H, et al. Quantitative intravoxel incoherent motion parameters derived from whole-tumor volume for assessing pathological complete response to neoadjuvant chemotherapy in locally advanced rectal cancer. J Mag Res Imag. 2018;48(1):248-258. doi:10.1002/jmri.25931

9. van der Sluis FJ, Couwenberg AM, de Bock GH, et al. Populationbased study of morbidity risk associated with pathological complete response after chemoradiotherapy for rectal cancer. $\mathrm{Br} J$ Surg. 2020;107(1):131-139. doi:10.1002/bjs.11324

10. Kreis ME, Ruppert R, Kube R, et al. MRI-based use of neoadjuvant chemoradiotherapy in rectal carcinoma: surgical quality and histopathological outcome of the OCUM Trial. Ann Surg Oncol. 2020;27 (2):417-427. doi:10.1245/s10434-019-07696-y

11. Maas M, Dijkhoff RAP, Beets-Tan R. Rectal cancer: assessing response to neoadjuvant therapy. Magn Reson Imaging Clin $\mathrm{N} \mathrm{Am}$. 2020;28(1):117-126. doi:10.1016/j.mric.2019.09.004

12. Suzuki C, Torkzad MR, Tanaka S, et al. The importance of rectal cancer MRI protocols on interpretation accuracy. World J Surg Oncol. 2008;6(1):89. doi:10.1186/1477-7819-6-89

13. Schurink NW, Lambregts DMJ, Beets-Tan RGH. Diffusion-weighted imaging in rectal cancer: current applications and future perspectives. Br J Radiol. 2019;92(1096):20180655. doi:10.1259/bjr.20180655 
14. Kalisz KR, Enzerra MD, Paspulati RM. MRI evaluation of the response of rectal cancer to neoadjuvant chemoradiation therapy. Radiographics. 2019;39(2):538-556. doi:10.1148/rg.2019180075

15. Feeney G, Sehgal R, Sheehan M, et al. Neoadjuvant radiotherapy for rectal cancer management. World J Gastroenterol. 2019;25 (33):4850-4869. doi:10.3748/wjg.v25.i33.4850

16. Kim S, Han K, Seo N, et al. T2-weighted signal intensity-selected volumetry for prediction of pathological complete response after preoperative chemoradiotherapy in locally advanced rectal cancer. Eur Radiol. 2018;28(12):5231-5240. doi:10.1007/s00330-018-5520-1

17. Neri E, Guidi E, Pancrazi F, et al. MRI tumor volume reduction rate vs tumor regression grade in the pre-operative re-staging of locally advanced rectal cancer after chemo-radiotherapy. Eur J Radiol. 2015;84(12):2438-2443. doi:10.1016/j.ejrad.2015.08.008

18. Park CH, Kim HC, Cho YB, et al. Predicting tumor response after preoperative chemoradiation using clinical parameters in rectal cancer. World J Gastroenterol. 2011;17(48):5310-5316. doi:10.3748/wjg.v17.i48.5310

19. Kim YC, Lim JS, Keum KC, et al. Comparison of diffusion-weighted MRI and MR volumetry in the evaluation of early treatment outcomes after preoperative chemoradiotherapy for locally advanced rectal cancer. J Mag Res Imag. 2011;34(3):570-576. doi:10.1002/ jmri.22696

20. Yu SK, Tait D, Chau I, Brown G. MRI predictive factors for tumor response in rectal cancer following neoadjuvant chemoradiation therapy-implications for induction chemotherapy? Int J Radiat Oncol Biol Phys. 2013;87(3):505-511. doi:10.1016/j.ijrobp.2013.06.2052

21. Das P, Skibber JM, Rodriguez-Bigas MA, et al. Predictors of tumor response and downstaging in patients who receive preoperative chemoradiation for rectal cancer. Cancer. 2007;109(9):1750-1755. doi:10.1002/cncr.22625

22. Armstrong D, Raissouni S, Price Hiller J, et al. Predictors of pathologic complete response after neoadjuvant treatment for rectal cancer: a Multicenter Study. Clin Colorectal Cancer. 2015;14(4):291-295. doi:10.1016/j.clcc.2015.06.001

23. Garland ML, Vather R, Bunkley N, Pearse M, Bissett IP. Clinical tumour size and nodal status predict pathologic complete response following neoadjuvant chemoradiotherapy for rectal cancer. Int J Colorectal Dis. 2014;29(3):301-307. doi:10.1007/s00384-0131821-7

24. Patel SV, Roxburgh CS, Vakiani E, et al. Distance to the anal verge is associated with pathologic complete response to neoadjuvant therapy in locally advanced rectal cancer. J Surg Oncol. 2016;114 (5):637-641. doi:10.1002/jso.24358

25. Bates DDB, Golia Pernicka JS, Fuqua JL 3rd, et al. Diagnostic accuracy of b800 and b1500 DWI-MRI of the pelvis to detect residual rectal adenocarcinoma: a multi-reader study. Abdoml Radiol. 2020;45(2):293-300. doi:10.1007/s00261-019-02283-x

26. Sathyakumar K, Chandramohan A, Masih D, Jesudasan MR, Pulimood A, Eapen A. Best MRI predictors of complete response to neoadjuvant chemoradiation in locally advanced rectal cancer. $\mathrm{Br}$ $J$ Radiol. 2016;89(1060):20150328. doi:10.1259/bjr.20150328

27. Lambregts DM, Rao SX, Sassen S, et al. MRI and diffusion-weighted mri volumetry for identification of complete tumor responders after preoperative chemoradiotherapy in patients with rectal cancer: a bi-institutional Validation Study. Ann Surg. 2015;262(6):1034-1039. doi:10.1097/SLA.0000000000000909

28. Yang L, Xia C, Liu D, et al. The role of readout-segmented echo-planar imaging-based diffusion-weighted imaging in evaluating tumor response of locally advanced rectal cancer after neoadjuvant chemoradiotherapy. Acta Radiol. 2020;61(9):1155-1164. doi:10.1177/0284185119897354

29. Horvat N, Veeraraghavan $H$, Khan M, et al. MR imaging of rectal cancer: radiomics analysis to assess treatment response after neoadjuvant therapy. Radiology. 2018;287(3):833-843. doi:10.1148/ radiol.2018172300
30. Choi MH, Oh SN, Rha SE, et al. Diffusion-weighted imaging: apparent diffusion coefficient histogram analysis for detecting pathologic complete response to chemoradiotherapy in locally advanced rectal cancer. J Mag Res Imag. 2016;44(1):212-220. doi:10.1002/ jmri.25117

31. Chidambaram V, Brierley JD, Cummings B, et al. Investigation of volumetric apparent diffusion coefficient histogram analysis for assessing complete response and clinical outcomes following pre-operative chemoradiation treatment for rectal carcinoma. Abdoml Radiol. 2017;42(5):1310-1318. doi:10.1007/s00261-016-1010-6

32. Kim SH, Lee JY, Lee JM, Han JK, Choi BI. Apparent diffusion coefficient for evaluating tumour response to neoadjuvant chemoradiation therapy for locally advanced rectal cancer. Eur Radiol. 2011;21(5):987-995. doi:10.1007/s00330-010-1989-y

33. Intven M, Reerink O, Philippens ME. Diffusion-weighted MRI in locally advanced rectal cancer: pathological response prediction after neo-adjuvant radiochemotherapy. Strahlenther Onkol. 2013;189 (2):117-122. doi:10.1007/s00066-012-0270-5

34. Blazic IM, Lilic GB, Gajic MM. Quantitative assessment of rectal cancer response to neoadjuvant combined chemotherapy and radiation therapy: comparison of three methods of positioning region of interest for ADC measurements at diffusion-weighted MR imaging. Radiology. 2016;282(2):614. doi:10.1148/radiol.2017164045

35. Enkhbaatar NE, Inoue S, Yamamuro H, et al. MR imaging with apparent diffusion coefficient histogram analysis: evaluation of locally advanced rectal cancer after chemotherapy and radiation therapy. Radiology. 2018;288(1):129-137. doi:10.1148/ radiol.2018171804

36. Lambrecht M, Vandecaveye V, De Keyzer F, et al. Value of diffusion-weighted magnetic resonance imaging for prediction and early assessment of response to neoadjuvant radiochemotherapy in rectal cancer: preliminary results. Int J Radiat Oncol Biol Phys. 2012;82(2):863-870. doi:10.1016/j.ijrobp.2010.12.063

37. Liang CY, Chen MD, Zhao XX, Yan CG, Mei YJ, Xu YK. Multiple mathematical models of diffusion-weighted magnetic resonance imaging combined with prognostic factors for assessing the response to neoadjuvant chemotherapy and radiation therapy in locally advanced rectal cancer. Eur J Radiol. 2019;110:249-255. doi:10.1016/j. ejrad.2018.12.005

38. Ha HI, Kim AY, Yu CS, Park SH, Ha HK. Locally advanced rectal cancer: diffusion-weighted MR tumour volumetry and the apparent diffusion coefficient for evaluating complete remission after preoperative chemoradiation therapy. Eur Radiol. 2013;23 (12):3345-3353. doi:10.1007/s00330-013-2936-5

39. Hu F, Tang W, Sun Y, et al. The value of diffusion kurtosis imaging in assessing pathological complete response to neoadjuvant chemoradiation therapy in rectal cancer: a comparison with conventional diffusion-weighted imaging. Oncotarget. 2017;8(43):75597-75606. doi:10.18632/oncotarget.17491

40. De Felice F, Magnante AL, Musio D, et al. Diffusion-weighted magnetic resonance imaging in locally advanced rectal cancer treated with neoadjuvant chemoradiotherapy. Eur J Surg Oncol. 2017;43 (7):1324-1329. doi:10.1016/j.ejso.2017.03.010

41. Curvo-Semedo L, Lambregts DM, Maas M, et al. Rectal cancer: assessment of complete response to preoperative combined radiation therapy with chemotherapy-conventional MR volumetry versus diffusion-weighted MR imaging. Radiology. 2011;260(3):734-743. doi:10.1148/radiol.11102467

42. Iima M, Le Bihan D. Clinical intravoxel incoherent motion and diffusion MR imaging: past, present, and future. Radiology. 2016;278(1):13-32. doi:10.1148/radiol.2015150244

43. Lu W, Jing $\mathrm{H}$, Ju-Mei $\mathrm{Z}$, et al. Intravoxel incoherent motion diffusion-weighted imaging for discriminating the pathological response to neoadjuvant chemoradiotherapy in locally advanced rectal cancer. Sci Rep. 2017;7(1):8496. doi:10.1038/s41598-017-09227-9 
44. Zhu HB, Zhang XY, Zhou XH, et al. Assessment of pathological complete response to preoperative chemoradiotherapy by means of multiple mathematical models of diffusion-weighted MRI in locally advanced rectal cancer: a prospective single-center study. J Mag Res Imag. 2017;46(1):175-183. doi:10.1002/jmri.25567

45. Fusco R, Sansone M, Granata V, et al. Diffusion and perfusion MR parameters to assess preoperative short-course radiotherapy response in locally advanced rectal cancer: a comparative explorative study among standardized index of shape by DCE-MRI, intravoxel incoherent motion- and diffusion kurtosis imaging-derived parameters. Abdoml Radiol. 2019;44(11):3683-3700. doi:10.1007/s00261-018-1801-z

46. Nougaret S, Vargas HA, Lakhman Y, et al. Intravoxel incoherent motion-derived histogram metrics for assessment of response after combined chemotherapy and radiation therapy in rectal cancer: initial experience and comparison between single-section and volumetric analyses. Radiology. 2016;280(2):446-454. doi:10.1148/ radiol.2016150702

47. Jensen JH, Helpern JA, Ramani A, Lu H, Kaczynski K. Diffusional kurtosis imaging: the quantification of non-gaussian water diffusion by means of magnetic resonance imaging. Magn Reson Med. 2005;53 (6):1432-1440. doi:10.1002/mrm.20508

48. Yu J, Xu Q, Song JC, et al. The value of diffusion kurtosis magnetic resonance imaging for assessing treatment response of neoadjuvant chemoradiotherapy in locally advanced rectal cancer. Eur Radiol. 2017;27(5):1848-1857. doi:10.1007/s00330-016-4529-6

49. Ciolina M, Caruso D, De Santis D, et al. Dynamic contrast-enhanced magnetic resonance imaging in locally advanced rectal cancer: role of perfusion parameters in the assessment of response to treatment. Radiol Med. 2019;124(5):331-338. doi:10.1007/s11547-018-0978-0

50. Tong T, Sun Y, Gollub MJ, et al. Dynamic contrast-enhanced MRI: use in predicting pathological complete response to neoadjuvant chemoradiation in locally advanced rectal cancer. $J$ Mag Res Imag. 2015;42(3):673-680.

51. Gollub MJ, Gultekin DH, Akin O, et al. Dynamic contrast enhanced-MRI for the detection of pathological complete response to neoadjuvant chemotherapy for locally advanced rectal cancer. Eur Radiol. 2012;22(4):821-831. doi:10.1007/s00330-011-2321-1

52. Zou H-H, Yu J, Wei Y, Wu J-F, Xu Q. Response to neoadjuvant chemoradiotherapy for locally advanced rectum cancer: texture analysis of dynamic contrast-enhanced MRI. J Mag Res Imag. 2019;49 (3):885-893.

53. Gollub MJ, Tong T, Weiser M, Zheng J, Gonen M, Zakian KL. Limited accuracy of DCE-MRI in identification of pathological complete responders after chemoradiotherapy treatment for rectal cancer. Eur Radiol. 2017;27(4):1605-1612. doi:10.1007/s00330-016-4493-1

54. De Cecco CN, Ganeshan B, Ciolina M, et al. Texture analysis as imaging biomarker of tumoral response to neoadjuvant chemoradiotherapy in rectal cancer patients studied with 3-T magnetic resonance. Invest Radiol. 2015;50(4):239-245. doi:10.1097/ RLI.0000000000000116
55. Aker M, Ganeshan B, Afaq A, Wan S, Groves AM, Arulampalam T. Magnetic resonance texture analysis in identifying complete pathological response to neoadjuvant treatment in locally advanced rectal cancer. Dis Colon Rectum. 2019;62(2):163-170. doi:10.1097/ DCR.0000000000001224

56. Yang L, Qiu M, Xia C, et al. Value of high-resolution DWI in combination with texture analysis for the evaluation of tumor response after preoperative chemoradiotherapy for locally advanced rectal cancer. AJR Am J Roentgenol. 2019;212(6):1-8. doi:10.2214/ AJR.18.20689

57. Liu Z, Zhang XY, Shi YJ, et al. Radiomics analysis for evaluation of pathological complete response to neoadjuvant chemoradiotherapy in locally advanced rectal cancer. Clin Cancer Res. 2017;23 (23):7253-7262. doi:10.1158/1078-0432.CCR-17-1038

58. Liu S, Wen L, Hou J, et al. Predicting the pathological response to chemoradiotherapy of non-mucinous rectal cancer using pretreatment texture features based on intravoxel incoherent motion diffusion-weighted imaging. Abdoml Radiol. 2019;44(8):2689-2698. doi:10.1007/s00261-019-02032-0

59. Cui Y, Yang X, Shi Z, et al. Radiomics analysis of multiparametric MRI for prediction of pathological complete response to neoadjuvant chemoradiotherapy in locally advanced rectal cancer. Eur Radiol. 2019;29(3):1211-1220. doi:10.1007/s00330-018-5683-9

60. Nie K, Shi L, Chen Q, et al. Rectal cancer: assessment of neoadjuvant chemoradiation outcome based on radiomics of multiparametric MRI. Clin Cancer Res. 2016;22(21):5256-5264. doi:10.1158/1078-0432. CCR-15-2997

61. Shi L, Zhang Y, Nie K, et al. Machine learning for prediction of chemoradiation therapy response in rectal cancer using pre-treatment and mid-radiation multi-parametric MRI. Magn Reson Imaging. 2019;61:33-40. doi:10.1016/j.mri.2019.05.003

62. Giannini V, Mazzetti S, Bertotto I, et al. Predicting locally advanced rectal cancer response to neoadjuvant therapy with (18)F-FDG PET and MRI radiomics features. Eur J Nucl Med Mol Imaging. 2019;46 (4):878-888. doi:10.1007/s00259-018-4250-6

63. van Griethuysen JJM, Lambregts DMJ, Trebeschi S, et al. Radiomics performs comparable to morphologic assessment by expert radiologists for prediction of response to neoadjuvant chemoradiotherapy on baseline staging MRI in rectal cancer. Abdoml Radiol. 2020;45 (3):632-643. doi:10.1007/s00261-019-02321-8

64. Zhang XY, Wang L, Zhu HT, et al. Predicting rectal cancer response to neoadjuvant chemoradiotherapy using deep learning of diffusion kurtosis MRI. Radiology. 2020;296(1):190936. doi:10.1148/ radiol.2020190936

65. Bibault JE, Giraud P, Housset M, et al. Deep learning and radiomics predict complete response after neo-adjuvant chemoradiation for locally advanced rectal cancer. Sci Rep. 2018;8(1):12611. doi:10.1038/s41598-018-30657-6

\section{Publish your work in this journal}

Cancer Management and Research is an international, peer-reviewed open access journal focusing on cancer research and the optimal use of preventative and integrated treatment interventions to achieve improved outcomes, enhanced survival and quality of life for the cancer patient.
The manuscript management system is completely online and includes a very quick and fair peer-review system, which is all easy to use. Visit http://www.dovepress.com/testimonials.php to read real quotes from published authors. 